\title{
Structural Information in Two-Dimensional Patterns: Entropy Convergence and Excess Entropy
}

\author{
David P. Feldman ${ }^{1,2}$, f and James P. Crutchfield ${ }^{2}$, f \\ ${ }^{1}$ College of the Atlantic, Bar Harbor, MA 04609 \\ ${ }^{2}$ Santa Fe Institute, 1399 Hyde Park Road, Santa Fe, NM 87501
}

(Dated: October 22, 2018)

\begin{abstract}
We develop information-theoretic measures of spatial structure and pattern in more than one dimension. As is well known, the entropy density of a two-dimensional configuration can be efficiently and accurately estimated via a converging sequence of conditional entropies. We show that the manner in which these conditional entropies converge to their asymptotic value serves as a measure of global correlation and structure for spatial systems in any dimension. We compare and contrast entropy-convergence with mutual-information and structure-factor techniques for quantifying and detecting spatial structure.

PACS: 05.20.-y 05.45.-a 65.40.Gr 89.70.+c 89.75.Kd

Santa Fe Institute Working Paper 02-12-06X arxiv.org/abs/cond-mat/0212XXX
\end{abstract}

\section{INTRODUCTION}

The past decade has seen considerable advances in our understanding of general ways to detect and quantify pattern in one-dimensional systems. This work is of intrinsic and general interest, since it suggests different ways of viewing patterns and calls attention to some of the subtleties associated with pattern discovery and quantification [1], issues that — implicitly or explicitly — underlie much of the scientific enterprise.

Recently, these abstract measures of structural complexity or pattern played a key role in several applications in physics and dynamical systems. For example, there is a growing body of work that seeks to relate the structural complexity of a one-dimensional sequence to the difficulty one encounters when trying to learn or synchronize to the generating process [2, 3, 4, 5]. Also, complexity measures have recently been used to characterize experimentally observed structures in a class of layered materials known as polytypes [6].

The successes in one dimension have not been readily followed by similar advances in two dimensions. Nonetheless, the development of a general measure of complexity - or pattern or structure - for two-dimensional systems is a longstanding goal. How is information shared, stored, and transmitted across a two-dimensional lattice to produce a given set of configurations? How can we quantitatively distinguish between different types of ordering or pattern in two dimensions? Though largely answered in one dimension, these questions are open in higher dimensions.

One oft-used set of techniques for examining patterns is Fourier or spectral analysis. This approach is well suited to detecting periodic ordering when the wavenumber of the transform matches the periodicity of the pattern.

\footnotetext{
*Electronic address: dpf@santafe.edu
}

${ }^{\dagger}$ Electronic address: chaos@santafe.edu
However, these methods typically rely on two-variable correlation functions. As such, they are incapable of distinguishing structures that differ in their correlations over more than two variables, as we shall see below.

Some recent work in this area, motivated in part by the need to characterize complex interfaces in surface science and geology 17, 8, 9, 10, 11, 12, 13, has suggested a set of approaches to these questions that are similar in spirit to fractal dimensions, in the sense that these approaches involve coarse-graining variables and then monitoring the changes that result as the coarse-graining scale is modulated. One can also use a multifractal approach, also known as the singularity spectrum, " $f(\alpha)$ ", the thermodynamic formalism, and the fluctuation spectrum; for reviews, see, e.g., 14, 15, 16. All of these approaches can be applied to spatial structures, but they suffer several drawbacks. For one, they are not fully spatial, in the sense that their calculation requires one to discard spatial information. Second, they do not directly speak to the correlation present in a system; rather they are more measures of entropy, disorder, and inhomogeneity.

Other recent general approaches to pattern in two dimensions include the extension of the formal theory of computation 17] and an information-theoretic approach [18 somewhat similar in spirit to that which we develop below. See also Ref. [19].

In this work, we take a different approach to the question of pattern and structure in two spatial dimensions. Our starting point is the excess entropy, an information-theoretic measure of complexity that is commonly used and well understood in one dimension [2, 20, 21, 22, 23, 24, 25, 26, 27, 28, 29]. Our main goals are severalfold. First, we introduce three ways to extend the definition of excess entropy to more than one dimension, noting that these extensions are not equivalent. Second, we report results of estimating two of these forms of excess entropy for a standard statistical mechanical system: the two-dimensional Ising model with nearestand next-nearest-neighbor interactions on a square lattice. We show that these two forms of excess entropy 
are similar but not identical, that each is sensitive to the structural changes the system undergoes, and that they are able to distinguish between different patterns that have the same structure factors. Third, we discuss some of the subtleties and challenges associated with moving from a one- to a two-dimensional information-theoretic analysis of pattern and structure.

\section{ENTROPY AND ENTROPY CONVERGENCE IN ONE DIMENSION}

We begin by reviewing information-theoretic quantities applied to one-dimensional (1D) systems. This allows us to define quantities and to fix notation that will be useful in our discussion of two-dimensional (2D) information theory in the subsequent section.

Let $X$ be a random variable that assumes the values $x \in \mathcal{X}$, where $\mathcal{X}$ is a finite set. We denote the probability that $X$ assumes the particular value $x$ by $\operatorname{Pr}(x)$. Likewise, let $Y$ be a random variable that assumes the values $y \in \mathcal{Y}$. The Shannon entropy of the random variable $X$ is defined by:

$$
H[X] \equiv-\sum_{x \in \mathcal{X}} \operatorname{Pr}(x) \log _{2} \operatorname{Pr}(x) .
$$

The entropy $H[X]$ measures the average uncertainty, in units of bits, associated with outcomes of $X$. The conditional entropy is defined by

$$
H[X \mid Y] \equiv-\sum_{x \in \mathcal{X}, y \in \mathcal{Y}} \operatorname{Pr}(x, y) \log _{2} \operatorname{Pr}(x \mid y)
$$

and measures the average uncertainty associated with variable $X$, if we know the outcome of $Y$. Finally, the mutual information between $X$ and $Y$ is defined as

$$
I[X ; Y] \equiv H[X]-H[X \mid Y] .
$$

Thus, $Y$ carries information about $X$ to the extent that knowledge of $Y$ reduces one's average uncertainty about $X$. The above three definitions are all standard; for details, see, e.g., Ref. 30].

\section{A. Block Entropy and Entropy Density}

Now consider a $1 \mathrm{D}$ chain $\ldots S_{-2} S_{-1} S_{0} S_{1} \ldots$ of random variables $S_{i}$ that range over a finite set $\mathcal{A}$. This chain may be viewed as a $1 \mathrm{D}$ spin system, a stationary time series of measurements, or an orbit of a symbolic dynamical system. We denote a block of $L$ consecutive variables by $S^{L} \equiv S_{1} \ldots S_{L}$. The probability that the particular $L$-block $s^{L}$ occurs is denoted $\operatorname{Pr}\left(s^{L}\right)$. We shall follow the convention that a capital letter refers to a random variable, while a lower case letter denotes a particular value of that variable.
We now examine the behavior of the Shannon entropy $H(L)$ of $S^{L}$. The total Shannon entropy of length- $L$ sequences - the block entropy -is defined by

$$
H(L)=-\sum_{s^{L} \in \mathcal{A}^{L}} \operatorname{Pr}\left(s^{L}\right) \log _{2} \operatorname{Pr}\left(s^{L}\right) .
$$

Graphically, we represent this as

$$
H(L) \equiv H[\overleftrightarrow{\square L \mid \square}]
$$

The sum in Eq. (4) is understood to run over all possible blocks of $L$ consecutive symbols. The entropy density is then defined as

$$
h_{\mu} \equiv \lim _{L \rightarrow \infty} \frac{H(L)}{L} .
$$

The above limit exists for all spatial-translation invariant systems [30]. Eqs. (6) and (4), together, are equivalent to the Gibbs entropy density. However, the informationtheoretic vantage point allows us to form another expression for the entropy density, one that will lead to a measure of structure.

The entropy density $h_{\mu}$ can be re-expressed as the limit of a form of conditional entropy. To do so, we first define

$$
h_{\mu}(L) \equiv H\left[S_{L} \mid S_{L-1} S_{L-2} \cdots S_{1}\right] .
$$

In words, $h_{\mu}(L)$ is the entropy of a single spin conditioned on a block of $L-1$ adjacent spins. This can also be written graphically:

$$
h_{\mu}(L)=H[\otimes \mid \stackrel{L-1 \longrightarrow}{\square !}]
$$

The pictogram on the right indicates that the entropy is conditioned on the $L-1$ spins directly to the right of the single target spin $\bigotimes$, with the bold vertical lines denoting the boundary where the target spin and spin block abut. One can then show that the entropy density defined in Eq. (6) can be written as:

$$
h_{\mu}=\lim _{L \rightarrow \infty} h_{\mu}(L) .
$$

For a proof that the limits in Eqs. (9) and (6) are equivalent, see Ref. 30]. As the block length $L$ grows, the terms in Eq. (9) typically converge to $h_{\mu}$ much faster than those in Eq. (6). See, e.g., Ref. 31 and citations therein.

\section{B. Excess Entropy}

The entropy density measures the randomness or unpredictability of the system; $h_{\mu}$ is the randomness that persists even after correlations over infinitely long blocks of variables are taken into account. A complementary quantity to the entropy density is the excess entropy $\mathbf{E}$ 20, 21, 22, 23, 24, 25, 26, 27, 28]. The excess entropy 
may be viewed as a measure of the apparent memory or structure in the system.

The excess entropy is defined by considering how the finite- $L$ entropy density estimates $h_{\mu}(L)$ converge to their asymptotic value $h_{\mu}$. For each $L$, the system appears more random than it actually is by an amount $h_{\mu}(L)-h_{\mu}$. Summing up these entropy-density overestimates gives us the excess entropy:

$$
\mathbf{E}_{\mathrm{C}} \equiv \sum_{L=1}^{\infty}\left(h_{\mu}(L)-h_{\mu}\right) .
$$

The excess entropy thus measures the amount of apparent randomness at small $L$ values that is "explained away" by considering correlations over larger and larger blocks. The subscript in $\mathbf{E}_{\mathrm{C}}$ indicates that this form of excess entropy is defined by considering how the entropy density converges to $h_{\mu}$.

Another expression for the excess entropy is obtained by looking at the growth of the block entropy $H(L)$. By Eq. (6), we know that $H(L)$ typically grows linearly for large $L$. The excess entropy can be shown to be equal to the portion of $H(L)$ that is sublinear- $\mathbf{E}$ is the subextensive part. That is, the excess entropy is defined implicitly by:

$$
H(L)=\mathbf{E}_{\mathrm{S}}+h_{\mu} L, \text { as } L \rightarrow \infty .
$$

Here, the subscript " $\mathrm{S}$ " on $\mathbf{E}_{\mathrm{S}}$ serves as a reminder that this expression for the excess entropy is the subextensive part of $H(L)$.

Finally, one can show [24, 29] that the excess entropy is also equal to the mutual information between two adjacent semi-infinite blocks of variables;

$$
\begin{aligned}
\mathbf{E}_{\mathrm{I}} & =\lim _{L \rightarrow \infty} I\left[S_{-L} \ldots S_{-2} S_{-1} ; S_{0} S_{1} \ldots S_{L-1}\right] \\
& =\lim _{L \rightarrow \infty} I[\square \stackrel{L}{\square-L} ; \square \longrightarrow \square] .
\end{aligned}
$$

The "I" in the subscript indicates that this expression for the excess entropy is given in terms of a mutual information. Note that in the pictographic version, Eq. (13), the two semi-infinite blocks are understood to be adjacent, as indicated by the thick vertical lines.

The three different forms for the excess entropy $-\mathbf{E}_{\mathrm{C}}$, $\mathbf{E}_{\mathrm{S}}$, and $\mathbf{E}_{\mathrm{I}}$ - given above are all equivalent in one dimension [24, 29]. We represent these different forms with distinct symbols because they are not identical in two dimensions.

In the subsequent section we compare our results for the excess entropies with various structure factorsstandard quantities from statistical physics used to detect periodic structure. The definition of the structure factor begins with the two-spin correlation function:

$$
\begin{aligned}
\Gamma_{i j} & \equiv\left\langle\left(s_{i}-\left\langle s_{i}\right\rangle\right)\left(s_{j}-\left\langle s_{j}\right\rangle\right)\right\rangle \\
& =\left\langle s_{i} s_{j}\right\rangle-\langle s\rangle^{2},
\end{aligned}
$$

where $s_{i}$ and $s_{j}$ denote the value of spins at different lattice coordinates. The second equality follows from the translation invariance of configurations. The angular brackets indicate a thermal expectation value. In $2 \mathrm{D}$ we will be interested in spins that are separated horizontally or vertically, but not both. (In a scattering scenario, this corresponds to restricting ourselves to a situation in which the particles to be scattered are incident along a line parallel to one of the axes of the lattice.) We define $\Gamma(r)$ as the correlation function between two spins separated, horizontally or vertically, by $r$ lattice sites:

$$
\Gamma(r) \equiv\left\langle s_{0} s_{r}\right\rangle-\langle s\rangle^{2} .
$$

The structure factor, then, is the discrete Fourier transform of the correlation functions:

$$
S(p)=\sum_{r=1}^{\infty} \cos \left(\frac{2 \pi r}{p}\right) \Gamma(r) .
$$

If the correlation function has a strong period- $p$ component, then $S(p)$ is large; if not, $S(p)$ is small. The absolute magnitude of $S(p)$ is generally not interpreted; only the relative change as a function of $p$ is. In this way, the structure factor serves as a signal of correlations in a configuration at a given periodicity.

It is widely held that the excess entropy $\mathbf{E}$ serves as a general purpose measure of a system's structure, regularity, or memory; for recent reviews, see [2, 26, 29]. The excess entropy provides a quantitative measure of structure that may be applied to any 1D symbolic string. In Refs. [28, 32, 33], we argued that $\mathbf{E}$ may be viewed as an effective order-parameter for 1D spin systems. In particular, we showed that the excess entropy is sensitive to periodic structure at any period, whereas structure factors, by construction, are sensitive to ordering at only a single spatial period. We shall return to this point below and show that the same general claim holds in two dimensions as well.

\section{TWO-DIMENSIONAL ENTROPY, ENTROPY DENSITY, AND EXCESS ENTROPY}

\section{A. Generalizing to Higher Spatial Dimensions}

Below we discuss how to extend the 1D analysis outlined above to apply to spatial patterns in two and higher dimensions. Before launching into definitions and formalism, we sketch some of the philosophy and intuitions that motivate the path we take and highlight some of the general issues that arise as one moves from $1 \mathrm{D}$ to $2 \mathrm{D}$ systems.

Patterns in two dimensions are fundamentally different than those in one dimension. For example, in one dimension a natural way to scan a configuration exists: left-to-right, say. That is, each local variable is indexed in a well defined order. (The information-theoretic measures discussed in the previous section have the same values regardless of whether the $1 \mathrm{D}$ configuration is scanned left-to-right or right-to-left.) 
The $1 \mathrm{D}$ approach simply does not generalize to $2 \mathrm{D}$ in such a unique, natural way. One might be tempted to scan or parse a $2 \mathrm{D}$ configuration by taking a particular 1D path through it. One would then apply 1D measures of randomness and structure to the sequences thus obtained. For example, in Refs. [34, 35], a space-filling curve is used to parse a $2 \mathrm{D}$ configuration and, from this, the entropy density of the configuration is estimated.

While the 1D-path method does yield the correct entropy density, it is also clear that it projects additional, spurious structure onto the configuration. By snaking through the lattice, it is inevitable that sites, adjacent in the 2D lattice, occur far apart in the $1 \mathrm{D}$ sequence. As a result, long-range correlations appear in the latter. Thus, a 1D excess entropy (or any other 1D measure of structural complexity) adapted in this way will capture not only properties of the $2 \mathrm{D}$ configuration, but also properties of the path. Except in special cases and with appropriate prior knowledge, it does not appear possible to disentangle these two distinct sources of apparent structure. These, and related difficulties with the 1D approach have been discussed in some detail in, for example, Refs. [22, 28, 36].

Here, we seek an alternative to understanding a $2 \mathrm{D}$ pattern by parsing it into $1 \mathrm{D}$ strings. We are immediately faced with a problem, however. There is a unique, complete ordering of the connected, nested subsets of a $1 \mathrm{D}$ lattice such that the conditional entropies of the target spin, conditioned on this sequence of subsets, are monotonic decreasing. It is this ordering that makes Eq. (10) unambiguous and unique in 1D. In contrast, connected, nested subsets of a 2D lattice that have this monotonic property are not unique. This is a direct consequence of the topological differences between one and two dimensional lattices. We shall see that this lack of uniqueness introduces ambiguity in extending Eq. (10) to two dimensions; specifically, there is no natural, unique expression for the excess entropy in two dimensions.

This lack of uniqueness is not a cause for concern. In fact, it seems a desirable property. Given the richness and subtleties of $2 \mathrm{D}$ patterns, one would expect that it would take more than one (or even several) complexity measures to adequately capture the range of $2 \mathrm{D}$ structures and orderings. These different measures will capture different features of the 2D configuration. As such, it is particularly important to specify the context in which a complexity measure is to be used and state what the measure is intended to capture, as we and others have argued elsewhere [27, 37, 38, 39].

As an example of this non-uniqueness in $2 \mathrm{D}$, consider what occurs when one moves from calculus of one variable to multi-dimensional calculus. In $1 \mathrm{D}$ calculus, the derivative is well defined for all smooth curves; the derivative is simply a number. In contrast, in $2 \mathrm{D}$ the derivative is not unique at each point on a surface; one must also specify the direction in which it is taken. There is a subspace (the tangent plane) of first derivatives of a smooth surface at any single point. A similar scenario appears to hold for the excess entropy in two dimensions. In Ref. [29] we synthesized a number of information-theoretic approaches to structure in one dimension by developing an analysis in terms of discrete derivatives and integrals. We expect that similar (although not unique) measures of structure, randomness, and memory can be developed for $2 \mathrm{D}$ systems by making use of discrete calculus in two dimensions. The work presented below is a first step in this direction.

\section{B. Entropy Density}

The entropy density in two dimensions is defined in the natural way. Consider an infinite $2 \mathrm{D}$ square lattice of random variables $S_{i j}$ whose values range over the finite set $\mathcal{A}$. Assuming that the variables are translationally invariant, the $2 \mathrm{D}$ entropy density is given by:

$$
h_{\mu}=\lim _{N, M \rightarrow \infty} \frac{H(N, M)}{N M},
$$

where $H(M, N)$ is the Shannon entropy of an $N$ x $M$ block of spin variables. This limit exists for a translationally invariant system, provided that the limits are taken in such a manner that the ration $N / M$ remains constant and finite.

Is there a way to re-express the 2D entropy density of Eq. (18) as the entropy of a target variable conditioned on a block of neighboring variables, analogous to Eq. (9)? This question was, to our knowledge, first answered in the affirmative by Alexandrowicz in the early 1970's 40, 41]. Meirovitch [42, 43] and later Schlijper and co-authors [44, 45] extended and applied Alexandrowicz's work. These methods have also been discovered independently by Eriksson and Lindgren 46, 47 and Olbrich et al. 48]. Here we briefly summarize the central result and adapt it to our needs.

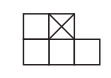

$$
\mathrm{M}=1
$$

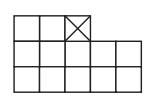

$$
M=2
$$

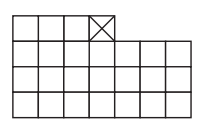

$\mathrm{M}=3$
FIG. 1: Neighborhood templates for 2D conditional entropies. The target spin is denoted with an X.

The most general approach to the conditional entropy in two dimensions proceeds as follows. Let $h_{\mu}(M)$ denote the Shannon entropy of the target spin conditioned on a $2 \mathrm{D}$ neighborhood template of $2 M(M+1)$ spins. Arrange the spin template in an $(M+1) \times(2 M+1)$ rectangle, with the target spin in the center of the rectangle's top row and with the top, rightmost $M$ spins deleted from the template. A sequence of neighborhood templates of this type is shown in Fig. 1. For example, $h_{\mu}(3)$ is the entropy of the target spin (denoted by an $\mathrm{X}$ ) conditioned on all the other spins in the rightmost template of Fig. 1. The 
$2 \mathrm{D}$ entropy density $h_{\mu}$ may then be shown to be equal to 46, 47, 49, 50]:

$$
h_{\mu}=\lim _{M \rightarrow \infty} h_{\mu}(M)
$$

If it is known that the interactions between spins are of finite range, then one only needs to use a shape as thick as the interaction range 40, 41, 44, 45, 46. For example, the following section we consider a $2 \mathrm{D}$ Ising model with nearest- and next-nearest-neighbor interactions. In this case, one uses a strip with a thickness of two lattice sites; see Fig. 2 .

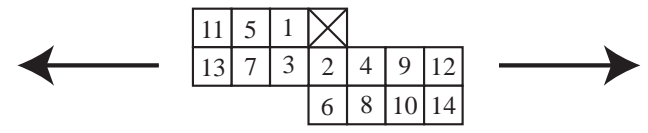

FIG. 2: Target spin (X) and neighborhood templates for conditional entropies used in our study of the 2D NNN Ising model. The cell numbers indicate the order in which the sites are added to the template. For more discussion, see text.

We now slightly modify the definition of the templatesize parameter $M$ in the conditional single-site entropy $h_{\mu}(M)$ so as to apply to the scenario in Fig. 2. The cell numbers in this figure indicate the order in which individual sites are added to the neighborhood template. For example, $h_{\mu}(3)$ now will denote the entropy of the target spin $\left(S_{00}\right)$ conditioned on the three spins labeled $1\left(S_{-10}\right), 2\left(S_{01}\right)$, and $3\left(S_{-11}\right)$; that is,

$$
h_{\mu}(3)=H\left[S_{00} \mid S_{-10}, S_{01}, S_{-11}\right] .
$$

In the $M \rightarrow \infty$ limit, the new $h_{\mu}(M)$ still goes to the entropy density, as in Eq. (19). It is also not hard to see that this convergence must be monotonic:

$$
h_{\mu}(M) \leq h_{\mu}\left(M^{\prime}\right), \quad M>M^{\prime} .
$$

This is a direct consequence of the fact that conditioning reduces entropy [30]; that is, the conditional entropy of a variable cannot increase as a result of increasing the number of variables upon which it is conditioned.

A few remarks about the neighborhood template in Fig. 2 are in order. First, the strip needs to be two sites thick since the system explored below has interactions that extend across two lattice sites. In this case, a strip with a thickness of two sites shields one half of the lattice from the other. In the limit that the strip is infinitely long in the horizontal direction, then the probability distribution of the target spin is independent of the values of the spins beneath the strip [50].

Second, at first blush, the numbering scheme in Fig. 2 appears ambiguous. Spins are added to the template in order of increasing Euclidean distance from the target spin. For example, spin 10 is a Euclidean distance $2 \sqrt{2}$ from the center spin, whereas spin 11 is a distance of 3 . Since $2 \sqrt{2}<3$, one adds on spin 10 before 11 . When there is a tie, one adds the leftmost spin. For example, spins 3 and 4 are the same Euclidean distance from the center spin; spin 3 comes before 4 since it is to the left.

Of course, one can use alternative ordering schemes, such as adding spins in a widening spiral or some other geometric pattern. These choices do not change the result in Eq. (19), since this is a statement about what happens in the limit that an arbitrarily large number of spins have been added to the template. However, looking ahead, the order in which spins are added can affect the convergence form of the $2 \mathrm{D}$ excess entropy - the $2 \mathrm{D}$ analog of $\mathbf{E}_{\mathrm{C}}$ of Eq. (10).

As noted above, the ambiguity in how the neighborhood template of conditioning variables grows is a direct result of the fact that a $2 \mathrm{D}$ lattice does not specify a strict ordering of its elements in the way that a $1 \mathrm{D}$ sequence does. Rather, a 2D lattice specifies a partial ordering of its elements. Thus, there will always be "ties" in the sense just mentioned, and so there is no unique, natural way to add on the spins one-by-one based on an ordering of subsets of spin blocks. See Ref. [51] for a detailed discussion of this, albeit in a slightly different context.

Third, there is a physical motivation for the neighborhood template of Fig. 2 articulated by Kikuchi [52. Picture a crystal growing by adsorbing one particle at a time. One can imagine that particles are added oneby-one, left to right, on top of already formed layers of the solid. This is exactly the process captured by the templates of Figs. 1 and 2 .

As remarked above, the conditional Shannon entropy method for calculating the entropy density $h_{\mu}$ is well known and has been successfully applied to a number of different systems. For example, in Ref. 45. Schlijper and Smit form upper and lower bounds for the entropy using block probabilities. They combine these bounds to obtain impressively accurate results for the entropy of the 2D Ising model and the $q=5,2 \mathrm{D}$ Potts model. This method for calculating the entropy has also been applied to the Ising model on a simple cubic lattice 43], a 2D hard-square lattice gas 53], the threedimensional fcc Ising antiferromagnet 54, coupled map lattices 48, Gaussian random fields 55], polymer chain models [56], and network-forming materials [57]. Quite recently, Meirovitch [56] estimated the entropy for the 2D Ising ferromagnet. Remarkably, his results have only a $0.01 \%$ relative error at the critical temperature, where one might expect the conditional entropy form to overestimate the entropy density due to long-range correlations missed by finite-size templates.

\section{Excess Entropy in Two Dimensions}

We now turn to the question of how to extend excess entropy to more than one dimension. In Sec. II B we saw that there were three different forms for the excess entropy: $\mathbf{E}_{\mathrm{C}}$, obtained by looking at how the entropy density converges to its asymptotic value; $\mathbf{E}_{\mathrm{I}}$, the excess entropy defined via a block-to-block mutual information; 
and $\mathbf{E}_{\mathrm{S}}$, the excess entropy as the subextensive part of the total entropy $H(L)$. In this section we consider three possible approaches to excess entropy in two dimensions. For each, we begin with one of the three different forms for the 1D excess entropy.

First, consider the convergence excess entropy $\mathbf{E}_{\mathrm{C}}$, as defined in Eq. (10). In the previous section we defined a sequence of $2 \mathrm{D}$ entropy density estimates $h_{\mu}(M)$ that converges from above to the entropy density $h_{\mu}$. We can sum these entropy density over-estimates to obtain the 2D convergence excess entropy:

$$
\mathbf{E}_{\mathrm{C}} \equiv \sum_{M=1}^{\infty}\left(h_{\mu}(M)-h_{\mu}\right)
$$

We shall see that this form of the excess entropy is, like its 1D cousin, capable of capturing the structures or correlations present in a 2D system. Note that this definition can depend on the order in which spins are added on to the template and, as discussed in the previous section, there is no unique ordering to use to determine the sequence in which to add sites. Nevertheless, our investigations have shown that any reasonable choice for ordering yields an $\mathbf{E}_{\mathrm{C}}$ that behaves qualitatively the same as that defined in Eq. (22).

The mutual information form $\mathbf{E}_{\mathrm{I}}$ of the excess entropy, defined in Eq. (13), can naturally be extended by considering the mutual information between two adjacent, infinite half-planes.

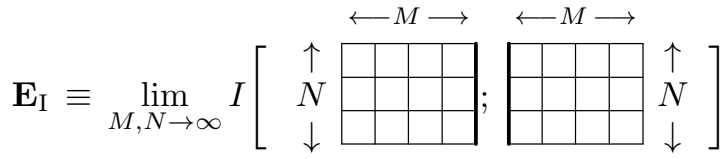

As in Eq. 13), it is understood that the two semi-infinite planes are adjacent.

Finally, one may also develop an expression for 2D subextensive excess entropies by considering how $H(M, N)$ grows with $M$ and $N$. In analogy to Eq. (11), we define three subextensive excess entropies via:

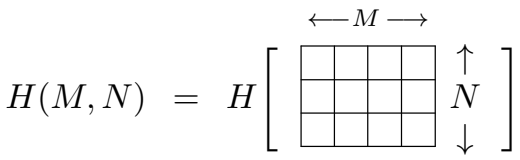

$$
\begin{aligned}
& \sim \mathbf{E}_{\mathrm{S}}+\mathbf{E}_{\mathrm{S}}^{x} M+\mathbf{E}_{\mathrm{S}}^{y} N+h_{\mu} M N .
\end{aligned}
$$

Note that in an isotropic system, such as that considered below, $\mathbf{E}_{\mathrm{S}}^{x}=\mathbf{E}_{\mathrm{S}}^{y}$. We shall not consider these forms for the 2D excess entropy here, opting instead to focus on $\mathbf{E}_{\mathrm{C}}$ and $\mathbf{E}_{\mathrm{I}}$.

\section{RESULTS}

\section{A. Next-Nearest-Neighbor Ising Systems}

To test the behavior of the different forms of the excess entropy, we estimated $\mathbf{E}_{\mathrm{I}}$ and $\mathbf{E}_{\mathrm{C}}$ numerically for a stan- dard system: the 2D spin-1/2 Ising model with nearestneighbor $(\mathrm{NN})$ and next-nearest-neighbor $(\mathrm{NNN})$ interactions. We choose this system since it is rich enough to exhibit several distinct structures and due to its broad familiarity. Its Hamiltonian $\mathcal{H}$ is given by:

$$
\begin{aligned}
\mathcal{H}= & -J_{1} \sum_{<i j, k l>\mathrm{nn}} S_{i j} S_{k l} \\
& -J_{2} \sum_{<i j, k l>\mathrm{nnn}} S_{i j} S_{k l}-B \sum_{i j} S_{i j},
\end{aligned}
$$

where the first (second) sum is understood to run over all NN (NNN) pairs of spins. Each spin $S_{i j}$ is a binary variable: $S_{i j} \in\{-1,+1\}$. The lattice consists of $N \times N$ spins; the spatial indices on spin variables run from 0 to $N-1$.

We estimated the structure factors $S(1), S(2)$, and $S(4)$ with Eq. (17) by directly measuring the frequency of occurrence of $s_{i} s_{j}$ and $s$ in spin configurations generated by a Monte Carlo simulation that used a standard single-site Metropolis algorithm on a lattice with periodic boundary conditions. That is, we sampled configurations with the canonical distribution: a configuration's probability is proportional to $e^{-\mathcal{H}(c) / T}$, where $\mathcal{H}(c)$ is the energy of the configuration $c$ and $T$ is the temperature. We used a lattice of $48 \times 48$ spins. Since we are not interested here in extracting the system's critical properties, there is no need to go to larger system sizes.

We estimated $\mathbf{E}_{\mathrm{C}}$ and $\mathbf{E}_{\mathrm{I}}$ from block probabilities by observing the frequency of spin-block occurrences. To estimate $\mathbf{E}_{\mathrm{C}}$ we used a template containing fifteen total spins, as shown in Fig. 2, and marginals of this distribution for smaller template sizes. To estimate $\mathbf{E}_{I}$ we calculated the mutual information of two adjacent $2 \times 4$ spin blocks. For each $J_{1}$ value we ran our Monte Carlo simulation for up to $2 \times 10^{5}$ Monte Carlo timesteps $\left(2 \times 10^{6}\right.$ for $\left.J_{1}<-1.5\right)$ and then took data every 20 timesteps for $2 \times 10^{4}$ timesteps. One Monte Carlo timestep corresponds to trying to flip, on average, each spin in the lattice one time. We thus sampled approximately $2 \times 10^{6}$ template configurations. For comparison, note that there are at most (in the highly disordered regime) $2^{16} \approx 3 \times 10^{4}$ possible configurations in a template of 16 spins.

\section{B. Excess Entropy Detects Periodic Structure}

Our results are shown in Fig. 3. The temperature was held at $T=1.0$, the external field at $B=0.0$, and the next-nearest-neighbor coupling at $J_{2}=-1.0$. Figure 3 shows $S(1), S(2), S(4), \mathbf{E}_{\mathrm{I}}$, and $\mathbf{E}_{\mathrm{C}}$, as a function of $J_{1} \in$ $[-4.0,4.0]$. For all $J_{1}$ values, the temperature is relatively small compared to the average energy per spin. And so, the configurations sampled are typically the ground state with a few low-energy excitations.

As $J_{1}$ is increased, the system moves through parameter regimes in which there are significant correlations of period 2, 4, and 1 . This is seen, for example, in the 

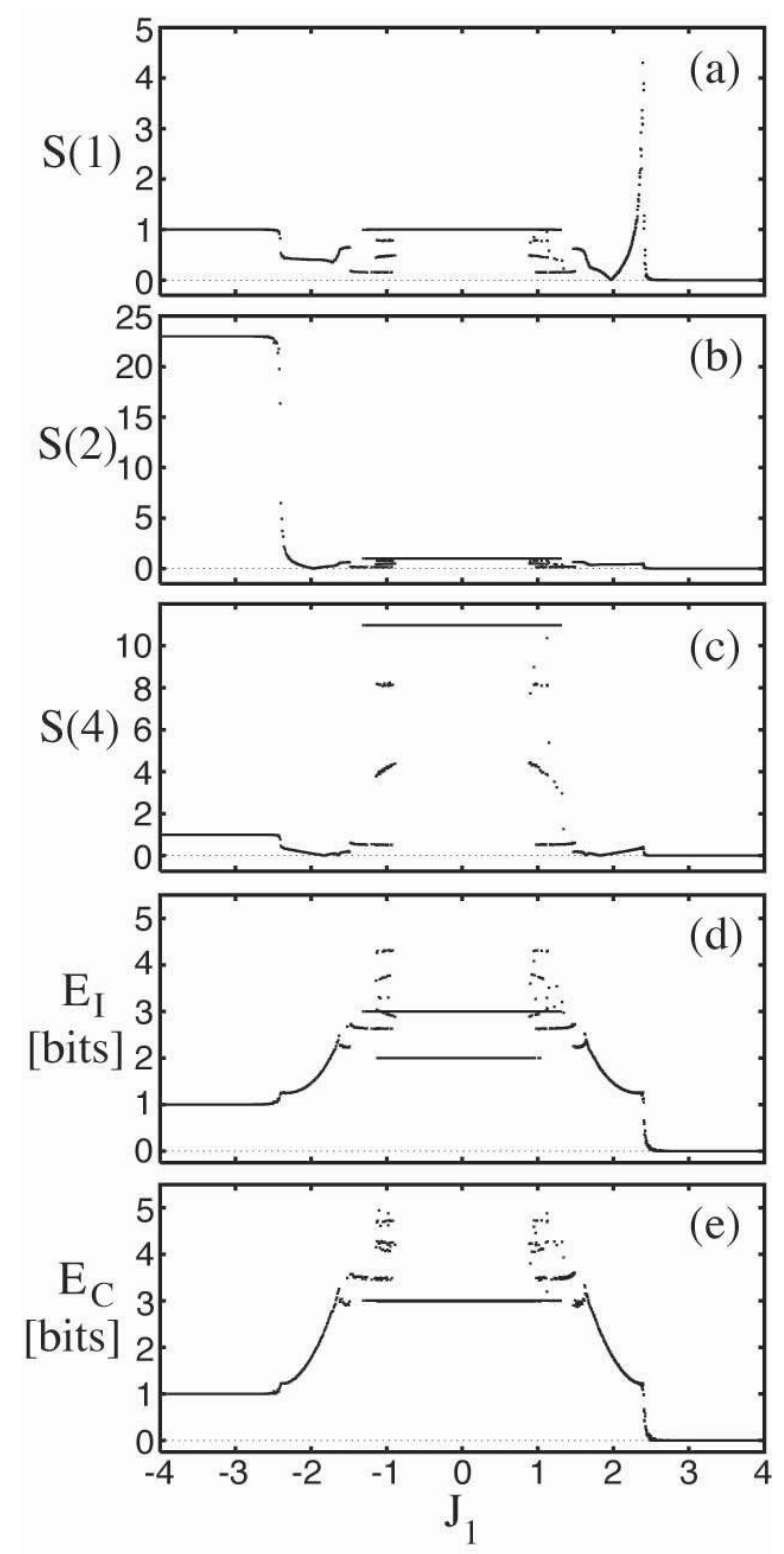

FIG. 3: Structural changes in the the 2D NNN Ising model as a function of NN coupling $J_{1}$ as revealed by structure factors (a) $S(1)$, (b) $S(2)$, and (c) $S(4)$, and excess entropies (d) $\mathbf{E}_{\mathrm{C}}$ (convergence) and (e) $\mathbf{E}_{\mathrm{I}}$ (mutual information). The temperature was fixed at $T=1.0$ and $J_{2}$ was held at -1.0 as the NN coupling was swept from $J_{1}=-4.0$ to $J_{1}=4.0$ in steps of $\delta J_{1}=0.01$, except near the $S(1)$ spike at $J_{1} \approx 2.5$ where $\delta J_{1}=0.005$. We performed at least 5 different runs at each $J_{1}$ in the range $\left|J_{1}\right| \leq 1.15$. Note the different scales on the vertical axes: the excess entropies are measured in bits of apparent memory; the structure factor magnitudes are arbitrary. For more discussion, see text.

behavior of the various structure factors; the structure factors selected correspond to periods of 2, 4, and 1 lattice sites.

Physically, when $J_{1}$ is large in magnitude and negative, the tendency for nearest neighbors to anti-align dominates and the system's ground state is antiferromag- netic: a checkerboard pattern consisting of alternating up and down spins. This pattern has a spatial period of 2. Not surprisingly, the period-2 structure factor $S(2)$ in Fig. 3(b) shows a strong signal in this low- $J_{1}$ regime.

When $J_{1}$ is near zero, the NN interactions are negligible compared to the NNN interactions. Thus, each spin orients opposite its four next-nearest neighbors, while disregarding its four nearest neighbors. The result is that the lattice effectively decouples into four, noninteracting sublattices. On each of these sublattices the spins alternate in sign, resulting in a ground state with spatial period 4 . Note that the period-4 structure factor $S(4)$ in Fig. 3(c) has a large value near $J_{1}=0$, indicating this period-4 ordering.

As $J_{1}$ is increased from 0 , the tendency for the spins to align grows stronger. Eventually this NN interaction overwhelms the NNN interactions and the entire lattice starts to align. This is the familiar paramagnetferromagnet transition. Above $J_{1} \approx 2.5$ the system acquires a net magnetization; there is now an unequal number of up and down spins, whereas below $J_{1} \approx 2.5$ there are always, on average, equal numbers of up and down spins. This transition is signaled by the distinct spike in the period-1 structure factor $S(1)$ near $J_{1} \approx 2.5$ and $S(1)$ 's vanishing at larger $J_{1}$. (The magnetic susceptibility $\chi$ diverges at the critical point of a ferromagnetparamagnet transition. Since $\chi \propto S(1)$, one expects to see a spike in $S(1)$ near this transition where the system acquires a non-zero magnetization.)

In Fig. 3(d) and 3(e) we plot the mutual-information excess entropy $\mathbf{E}_{\mathrm{I}}$ and the convergence excess entropy $\mathbf{E}_{\mathrm{C}}$ versus $J_{1}$ over the same parameter range. In the large and negative $J_{1}$ regime $\mathbf{E}_{\mathrm{I}}=\mathbf{E}_{\mathrm{C}}=1$ bit, indicating that there is one bit of information stored in the configurations. The configurations have a simple structure (alternating up-down spins) and the magnitude of $\mathbf{E}$ gives the information needed to specify the spatial phase of the period- 2 configurations. When $J_{1}$ is large and the system undergoes the transition to ferromagnetic ordering, $\mathbf{E}_{\mathrm{I}}=\mathbf{E}_{\mathrm{C}}=0$, since the configurations consist of all aligned spins, and there is no spatial information or structure in them. In the intermediate regime $\left(J_{1} \approx 0\right)$, $\mathbf{E}_{\mathrm{I}}$ and $\mathbf{E}_{\mathrm{C}}$ are markedly larger, indicating that the system is more structured than elsewhere. We will return shortly to discuss in detail what the values of $\mathbf{E}_{\mathrm{I}}$ and $\mathbf{E}_{\mathrm{C}}$ mean.

Note that each excess entropy is sensitive to correlations at all periodicities, despite the fact that each is merely a single, unparameterized function. In contrast, the structure factors $S(p)$ are a one-parameter family of functions that must be tuned a posteriori to find relevant periodic structure. That is, the period-1 structure factor $S(1)$ detects only the period-1 correlations near $J_{1}=2.5$. Moreover, $S(1)$ is unable to distinguish between the period- 2 and period- 4 orderings at $J_{1}<-3.0$ and $J_{1} \approx 0$, respectively; $S(1) \approx 1$ for both period- 2 and period-4 configurations.

Since the excess entropy $\mathbf{E}$ is a single, unparameterized 
function sensitive to structure of any periodicity, it is a more general measure of structure and correlation than the structure factors $S(p)$. Conversely, $S(p)$ is somewhat myopic. By considering only two-point correlations modulated at a selected periodicity $p, S(p)$ misses structure that is either aperiodic or that is due to more-than-twospin correlations. In fact, $\mathbf{E}$ is even more sensitive and general that these observations indicate.

\section{E Distinguishes Structurally Distinct Ground States}

Looking closely at the mutual-information excess entropy $\mathbf{E}_{\mathrm{I}}$ near $J_{1}=0$ in Fig. $3(\mathrm{~d})$, one notices that the curve splits in two in the $\left|J_{1}\right|<1.0$ region. This can be seen more clearly in Fig. $\mathbb{A}$, in which we plot $\mathbf{E}_{\mathrm{I}}$ versus $J_{1}$ in this region. We sampled the NN coupling $J_{1}$ every 0.01 and we performed at least five different runs at each $J_{1}$ value. Sometimes $\mathbf{E}_{\mathrm{I}}=3.0 \mathrm{bits}$, whereas for other trials $\mathbf{E}_{\mathrm{I}}=2.0$ bits. Why are there two different values for $\mathbf{E}_{\mathrm{I}}$ on different runs? And why, in contrast, is the period-4 structure factor $S(4)$ the same for all runs?

The answer is simple: there are multiple structurally distinct ground states. The three possible ground-state configurations are shown in Fig. F. Note that for each ground state, all NNN pairs of sites have opposite spin values, thus minimizing the system's energy. Note also that each ground state is identical if one considers only a horizontal or vertical slice; the repeating pattern of two up spins followed by two down spins is the same.

After a long transient time, the system usually settles into one of these three states. A boundary defect between two different ground states has an energy cost associated with it. As such, most boundaries are eventually destroyed. Incidentally, the dynamics through which this removal of boundary defects occurs is rather subtle and can be very long-lived. For example, a boundary between left and right diagonal phases costs more than a boundary between the checkerboard and one of the striped patterns. As a result, when the two different striped phases come close, the checkerboard pattern emerges between them, pushing the stripe boundaries away from each other. Moreover, as the temperature approaches zero, we observe that there are times when the ground state is simply not found via single-flip Metropolis Monte Carlo dynamics. Similar phenomena have been observed in other antiferromagnetic Ising models; for recent work, see Refs. [58, 59, 60].

In any event, a straightforward calculation shows that $\mathbf{E}_{\mathrm{I}}=3$ bits for the checkerboard configuration of Fig. (a), whereas $\mathbf{E}_{\mathrm{I}}=2$ bits for the two striped phases. (Similar calculations show that $\mathbf{E}_{\mathrm{C}}=3$ bits for both the checkerboard and striped ground states.) Note, however, that $S(4)$ is the same for all three ground states. By construction, $S(4)$ measures only two-spin statistics obtained by considering correlations along a horizontal or a vertical direction. And so, the three ground states are

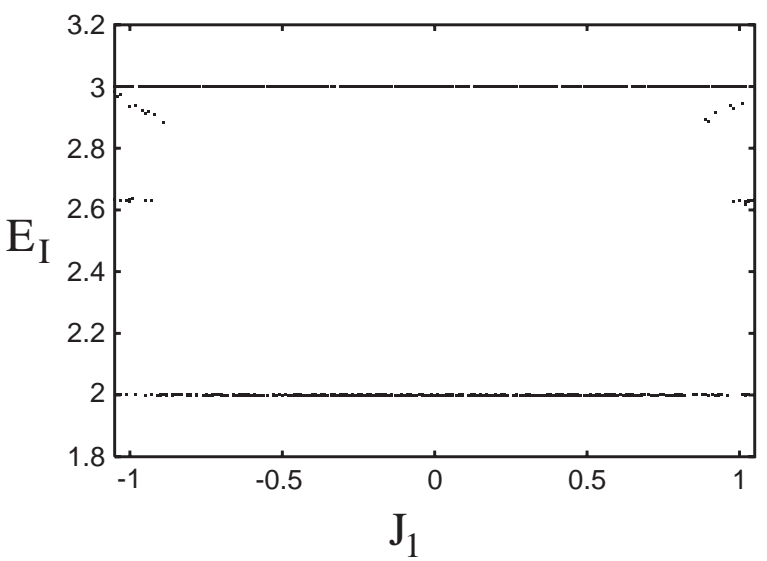

FIG. 4: The mutual-information excess entropy $\mathbf{E}_{\mathrm{I}}$ showing the existence of multiple period- 4 ground states.

the same if one considers only isolated horizontal or vertical slices; every slice consists of a repeating pattern of two up spins followed by two down spins. Of course, one can adapt the definition of $S(p)$ to account for the diagonal striped phases, but this simply begs the question of discovering the intrinsic patterns in the first place.

Near $\left|J_{1}\right|=1$ notice that $\mathbf{E}_{\mathrm{I}}$ and $\mathbf{E}_{\mathrm{C}}$ occur in plateaus between 2 and 3 bits and above. This indicates that the system has settled into a number of more structured metastable states consisting of mixtures of the three ground states.

In summary, we see that the mutual information excess entropy $\mathbf{E}_{\mathrm{I}}$ is capable of distinguishing between patterns that are not distinct according to the structure factors $S(p)$. In fact, we initially did not anticipate the two striped ground states, glibly assuming that the only ground state is the checkerboard. Our results for $\mathbf{E}_{\mathrm{I}}$, which we initially found confusing, led us to examine the configurations more closely and to detect the distinct ground state structures. This, in turn, led us to notice the rich dynamics of the configurations as they wend their way towards one of the three ground states. In short, these structural subtleties would have been missed entirely had we relied solely on the structure factors.

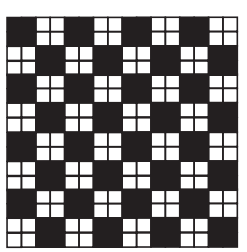

(a)

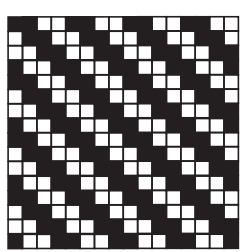

(b)

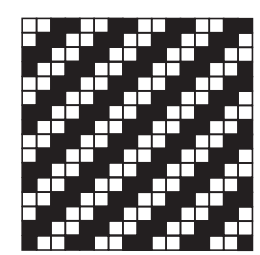

(c)
FIG. 5: The three ground states for $J_{1} \approx 0, J_{2}<0$ : (a) checker board, (b) left-diagonal stripe, and (c) right-diagonal stripe. 


\section{DISCUSSION AND CONCLUSION}

We have introduced three extensions of the excess entropy that apply to two-dimensional configurations. Each excess entropy expression is based on a different way of viewing the one-dimensional excess entropy: the convergence excess entropy $\mathbf{E}_{\mathrm{C}}$ measures the manner in which finite-template entropy density estimates converge to their asymptotic value; the subextensive excess entropy $\mathbf{E}_{\mathrm{S}}$ is related to the subextensive forms of the block entropy $H(M, N)$; and the mutual information excess entropy $\mathbf{E}_{\mathrm{I}}$, is defined as the mutual information between two halves of a configuration.

Applying two of these measures, $\mathbf{E}_{\mathrm{C}}$ and $\mathbf{E}_{\mathrm{I}}$, to the NNN Ising model, we have seen that these quantities capture the structural changes this system undergoes as its parameters are varied. In contrast, the structure factors are sensitive to periodic ordering of a particular period. Moreover, our results show that the information excess entropy $\mathbf{E}_{\mathrm{I}}$ cleanly distinguishes between two period-4 ground states, whereas the period-4 structure factor is simply incapable of making such a structural distinction. Finally, the values that the excess entropies take on are interpretable and give a quantitative measure of the amount of structure in the system.

The picture that emerges, then, is that the various twodimensional excess entropies behave as expected; they are clearly general purpose measures of two-dimensional structure. The excess entropy, being sensitive to multispin correlations, is capable of capturing patterns that a particular structure factor misses. The excess entropy does not decompose a pattern into periodic components, reporting instead a measure of the total amount of apparent information in a system.

The goal of this work is not to suggest that the excess entropy replace structure factors or, more generally, Fourier analysis. We view the excess entropy not in competition with Fourier analysis, but complementary to it; the excess entropy is designed to answer a different set of questions than those addressed by Fourier components. For example, it has long been appreciated in dynamical systems that power spectral analysis is of little help in revealing the geometry of a chaotic attractor [61]. Analogously, spectral decomposition typically will say little about how difficult it is to learn or synchronize to a pattern.

Clearly, however, there is much more work to be done to develop a thorough, well understood methodology for two-dimensional patterns. One possible approach builds on Refs. 44, 29, 62 which take a systematic look at entropy growth and convergence by using a discrete calculus. This work places several complexity measures within a common framework and leads to new measures of structure. From the study presented above, we conclude that a similar analysis in two dimensions, using a two-dimensional discrete calculus, holds great promise.
Another area for future research concerns developing relationships between measures of complexity of a pattern and the difficulty of learning or synchronizing to it. There has been recent work on this in one dimension [2, 3, 4. For example, in Refs. [4] we showed that the transient information [29], an information-theoretic quantity complementary to the excess entropy, measures the total uncertainty experienced by an observer who, given an accurate model of a process, must synchronize to it. Synchronization, in this sense, means determining with certainty in which internal state the process is. Establishing a similar result in $2 \mathrm{D}$ would be a significant aid in understanding new aspects of higher-dimensional patterns.

There are also, of course, a host of additional statistical mechanical systems, each with its own range of distinct structures, that should be similarly analyzed. Calculating excess entropies for them will facilitate developing our understanding of the behavior of these different quantities and may even lead to discovering novel structural properties. A natural choice is calculating the behavior of $\mathbf{E}$ near the critical temperature, extracting critical exponents, and relating these exponents to others for the well studied nearest-neighbor Ising model. It will also be of interest to calculate the various excess entropy forms for noisy Sierpinsky carpets and the like; this will allow for direct comparison with calculations of the measures of inhomogeneity put forth in Refs. [11, 12.

Ultimately, these different measures of structure those presented here and those developed by other authors - will be judged not solely by their ability to shed light on existing, well understood model systems such as the NNN Ising model considered here. Instead, the broader concern is how to use these informationtheoretic quantities to capture structure and patterns in systems that are less well understood. Equally important is the question of establishing relationships between information-theoretic measures of structural complexity and other quantities, including: physical measures of structure and correlation; computation-theoretic properties; and the difficulty of learning a pattern.

\section{Acknowledgments}

We thank Kristian Lindgren, Susan McKay, and Karl Young for helpful discussions. This work was supported at the Santa Fe Institute under the Computation, Dynamics, and Inference Program via SFI's core grants from the National Science and MacArthur Foundations. Direct support was provided from DARPA contract F30602-00-2-0583. DPF thanks the Department of Physics and Astronomy at the University of Maine for their hospitality. The Linux cluster used for the simulations reported here was provided by Intel Corporation through its support of SFI's Network Dynamics Program. 
[1] J. P. Crutchfield. The calculi of emergence: Computation, dynamics, and induction. Physica D, 75:11-54, 1994.

[2] W. Bialek, I. Nemenman, and N. Tishby. Predictability, complexity, and learning. Neural Computation, 13:24092463, 2001.

[3] I. Nemenman. Information Theory and Learning: A Physical Approach. PhD thesis, Princeton University, 2000.

[4] J. P. Crutchfield and D. P. Feldman. Synchronizing to the environment: Information theoretic constraints on agent learning. Advances in Complex Systems, 4:251-264, 2001.

[5] E. Dȩbowski. Entropic subextensitivity in language and learning. In C. Tsallis and M. Gell-Mann, editors, Nonextensive Entropy: Interdisciplinary Applications. Oxford University Press, 2002.

[6] D. P. Varn, G. S. Canright, and J. P. Crutchfield. Discovering planar disorder in close-packed structures from X-ray diffraction: Beyond the fault model. Physical Review B, 2002. In Press.

[7] C. Andraud, A. Beghdadi, and J. Lafait. Entropic analysis of random morphologies. Physica A, 207:208-212, 1994.

[8] C. Andraud, A. Beghdadi, E. Haslund, R. Hilfer, J. Lafait, and B. Virgin. Local entropy characterization of correlated random microstructures. Physica A, 235:307-318, 1997.

[9] C. DeW. Van Siclen. Information entropy of complex structures. Phys. Rev. E, 56:5211-15, 1997.

[10] R. Piasecki. Entropic measure of spatial disorder for systems of finite-sized objects. Physica A, 277:157-73, 2000.

[11] R. Piasecki. Detecting self-similarity in surfaces microstructures. Surface Science, 454-456:1058-1062, 2000.

[12] R. Piasecki, M. T. Martin, and A. Plastino. Inhomogeneity and complexity measures for spatial patterns. Physica A, 307, 2002.

[13] A. N. Pavlov, W. Ebeling, L. Molgedey, A. R. Ziganshin, and V. S. Anishchenko. Scaling features of tests, images and time series. Physica A, 300:310-324, 2001.

[14] K. Young and J. P. Crutchfield. Fluctuation spectroscopy. Chaos, Solitons, and Fractals, 4:5-39, 1993.

[15] J. L. McCauley. Introduction to multifractals in dynamical systems theory and fully developed fluid turbulence. Physics Reports, 189:225-266, 1990.

[16] C. Beck and F. Schlögl. Thermodynamics of Chaotic Systems. Cambridge University Press, 1993.

[17] K. Lindgren, C. Moore, and M. G. Nordahl. Complexity of two-dimensional patterns. J. Stat. Phys., 91:909-951, 1998.

[18] Y. A. Andrienko, N. V. Brilliantov, and J. Kurths. Complexity of two-dimensional patterns. Europ. Phys. J. B, 15:539-546, 2000.

[19] E. M. Sal'nikova and L. M. Martyushev. Determining the order parameter for the morphological analysis of two-dimensional structures. Technical Physics Letters, 27:301-304, 2001.

[20] J. P. Crutchfield and N. H. Packard. Symbolic dynamics of noisy chaos. Physica D, 7:201-223, 1983.

[21] R. Shaw. The Dripping Faucet as a Model Chaotic System. Aerial Press, Santa Cruz, California, 1984.

[22] P. Grassberger. Toward a quantitative theory of self- generated complexity. Intl. J. Theo. Phys., 25(9):907938, 1986.

[23] K. Lindgren and M. G. Norhdal. Complexity measures and cellular automata. Complex Systems, 2(4):409-440, 1988.

[24] W. Li. On the relationship between complexity and entropy for Markov chains and regular languages. Complex Systems, 5(4):381-399, 1991.

[25] D. Arnold. Information-theoretic analysis of phase transitions. Complex Systems, 10:143-155, 1996.

[26] W. Ebeling. Prediction and entropy of nonlinear dynamical systems and symbolic sequences with LRO. Physica D, 109:42-52, 1997.

[27] D. P. Feldman and J. P. Crutchfield. Measures of statistical complexity: Why? Physics Letters A, 238:244-252, 1998.

[28] D. P. Feldman. Computational Mechanics of Classical Spin Systems. PhD thesis, University of California, Davis, 1998.

[29] J. P. Crutchfield and D. P. Feldman. Regularities unseen, randomness observed: Levels of entropy convergence. Chaos, 2003. In Press.

[30] T. M. Cover and J. A. Thomas. Elements of Information Theory. John Wiley \& Sons, Inc., 1991.

[31] T. Schürmann and P. Grassberger. Entropy estimation of symbol sequences. Chaos, 6:414-427, 1996.

[32] J. P. Crutchfield and D. P. Feldman. Statistical complexity of simple one-dimensional spin systems. Phys. Rev. E, 55(2):1239R-1243R, 1997.

[33] D. P. Feldman and J. P. Crutchfield. Discovering noncritical organization: Statistical mechanical, information theoretic, and computational views of patterns in simple one-dimensional spin systems. 1998. Santa Fe Institute Working Paper 98-04-026.

[34] A. Lempel and J. Ziv. Compression of two-dimensional data. IEEE Transactions on Information Theory, 32:2-8, 1986.

[35] D. Sheinwald, A. Lempel, and J. Ziv. Two-dimensional encoding by finite-state encoders. IEEE Transactions on Information Theory, 1990.

[36] E. Soljanin. Writing sequences on the plane. IEEE Transactions on Information Theory, 48:1344-1354, 2002.

[37] C. H. Bennett. How to define complexity in physics, and why. In W. H. Zurek, editor, Complexity, Entropy, and the Physics of Information, volume VIII of Santa Fe Institute Studies in the Sciences of Complexity, pages 137-148. Addison-Wesley, 1990.

[38] P. M. Binder. Comment II on Simple Measure for Complexity. Phys. Rev. E, 62:2998-9, 2000.

[39] J. P. Crutchfield, D. P. Feldman, and C. R. Shalizi. Comment I on Simple Measure for Complexity. Phys. Rev. E, 62:2996-7, 2000.

[40] Z. Alexandrowicz. Stochastic models for the statistical description of lattice systems. J. Chem. Phys., 55:27652779, 1971.

[41] Z. Alexandrowicz. Entropy calculated from the frequency of states of individual particles. J. Stat. Phys., 14:1-9, 1976.

[42] H. Meirovitch. Calculation of entropy with computer simulation methods. Chem. Phys. Lett., 45:389-392, 1977.

[43] H. Meirovitch. Methods for estimating entropy with com- 
puter simulation: the simple cubic Ising lattice. J. Phys. A, 16:839-848, 1983.

[44] A. G. Schlijper, A. R. D. van Bergen, and B. Smit. Local-states method for the calculation of free energies in Monte Carlo simulations of lattice models. Phys. Rev. A, 41:1175-78, 1990.

[45] A. G. Schlijper and B. Smit. Two-sided bounds on the free energy from local states in Monte Carlo simulations. J. Stat. Phys., 56:247-60, 1989.

[46] K.-E. Eriksson and K. Lindgren. Entropy and correlations in lattice systems. Technical report, Physical Resource Theory Group, Chalmers University of Technology and University of Göteborg, 1989.

[47] K. Lindgren. Entropy and correlations in discrete dynamical systems. In J. L. Casti and A. Karlqvist, editors, Beyond Belief: Randomness, Prediction and Explanation in Science, pages 88-109. CRC Press, Boca Raton, Florida, 1991.

[48] E. Olbrich, R. Hegger, and H. Kantz. Local estimates for entropy densities in coupled map lattices. Phys. Rev. Lett., 84:2132-5, 2000.

[49] A. G. Schlijper. Convergence of the cluster-variation method in the thermodynamic limit. Phys. Rev. B, 27:6841-48, 1983.

[50] S. Goldstein, R. Kuik, and A. G. Schlijper. Entropy and global Markov properties. Comm. Math. Phys., 128:46982, 1990.

[51] B. Baumgartner. A partial ordering of sets, making mean entropy monotone. J. Phys. A, 35:3163-3182, 2002.

[52] R. Kikuchi. Crystal-growth approach of lattice-order simulation and the cluster-variation method. Phys. Rev. B,
22:3784-3789, 1980.

[53] H. Meirovitch. A Monte Carlo study of the entropy, the pressure, and the critical behavior of the hard-square lattice gas. Journal of Statistical Physics, 30:681-698, 1983.

[54] H. Meirovitch. Computer simulation study of hysteresis and free energy in the fcc Ising antiferromagnet. Physical Review B, 30:2866-2874, 1984.

[55] S. Marcelja. Entropy of phase-separated structures. Physica A, 231:168-177, 1996.

[56] H. Meirovitch. Simulation of a free energy upper bound, based on the anticorrelation between an approximate free energy functional and its fluctuation. Journal of Chemical Physics, 1111:7215-24, 199.

[57] R. L. C. Vink and G. T. Barkema. Configurational entropy of network-forming materials. arXiv:condmat/0204128, 2002.

[58] V. Spirin, P.L. Krapivsky, and S. Redner. Fate of zero-temperature "Ising ferromagnets. Phys. Rev. E, 63:036118, 2001.

[59] V. Spirin, P.L. Krapivsky, and S. Redner. Freezing in Ising ferromagnets. Phys. Rev. E, 63:016119, 2001.

[60] F. Vazquez, P. L. Krapivsky, and S. Redner. Freezing and slow evolution in a constrained opinion dynamics model. cond-mat/0209445, 2002.

[61] J. D. Farmer, J. P. Crutchfield, H. Froehling, N. H. Packard, and R. S. Shaw. Power spectra and mixing properties of strange attractors. Annals of the New York Academy of Sciences, 357:453, 1980.

[62] P.-M. Binder and J. A. Plazas. Multiscale analysis of complex systems. Phys. Rev. E, 63:065203(R), 2001. 